\title{
Infarto agudo del miocardio, una manifestación poco frecuente en la granulomatosis con poliangeítis
}

\section{Acute myocardial infarction, as a rare manifestation in granulomatosis with polyangiitis}

Lucía V. Maya-Piña ${ }^{1 *}$, Rosa E. Barbosa-Cobos ${ }^{1}$, Gustavo E. Lugo-Zamudio², Mª Elena

Cassaigne-Guasco ${ }^{3}$, Rubén Nicolás-Mendoza y Laura E. Godínez-Baca ${ }^{1}$

'Servicio de Reumatología, Hospital Juárez de México; ²Departamento de Dirección, Hospital Juárez de México; ${ }^{3}$ Servicio de Cardiología, Centro Médico ABC; ${ }^{4}$ Servicio de Cardiología, Hospital Juárez de México. Ciudad de México, México

\section{Resumen}

La granulomatosis con poliangeítis es una vasculitis de pequeños vasos asociada a la presencia de anticuerpos anticitoplasma de neutrófilos, con manifestaciones cardíacas que son poco frecuentes, como pericarditis, miocarditis, arteritis coronaria y enfermedad valvular. Reportamos el caso de un paciente de 49 años con reciente diagnóstico de granulomatosis con poliangeítis, quien presentó infarto agudo del miocardio. Se consideró la actividad de la enfermedad como causa del infarto. Las manifestaciones clínicas cardiovasculares en la granulomatosis con poliangeítis son relevantes por ser marcadores de mal pronóstico.

Palabras clave: Arteritis coronaria. Enfermedad cardíaca. Granulomatosis con poliangeítis. Involucro cardíaco. Manifestaciones cardíacas.

\begin{abstract}
Granulomatosis with polyangiitis is a small vessel vasculitis associated to anti-neutrophil cytoplasmic antibodies, in which the cardiac manifestations are not common, as pericarditis, cardiomyopathy, coronary artery disease and vascular disease. We report a clinical case of a 49-year-old man with a recent diagnosis of granulomatosis with polyangiitis, he presented myocardial infarction. Disease activity was considered the cause of myocardial infarction. Cardiovascular clinical manifestations in granulomatosis with polyangiitis are relevant because are markers of poor prognosis.
\end{abstract}

Key words: Coronary arteritis. Cardiac disease. Granulomatosis with polyangiitis. Cardiac involvement. Cardiac manifestations.

\section{Introducción}

La granulomatosis con poliangeítis es una vasculitis de pequeños vasos, necrosante, granulomatosa, asociada a anticuerpos anticitoplasma de neutrófilos
(ANCA), que afecta principalmente la vía respiratoria alta, el oído y la garganta, hasta en el $73-83 \%$ de los $\operatorname{casos}^{1,2}$.

El involucro cardíaco se presenta en el $3.3-10 \%$ de los $\operatorname{casos}^{2,3}$. La prevalencia de las manifestaciones cardíacas varía según el método de evaluación

\footnotetext{
Correspondencia:

*Lucía V. Maya-Piña

Av. Instituto Politécnico Nacional, 5160

Col. Magdalena de las Salinas, Alcaldía Gustavo A. Madero Fecha de recepción: 18-12-2019

C.P. 06770, Ciudad de México, México Fecha de aceptación: 01-04-2020

E-mail: luvemap @ hotmail.com

DOI: $10.24875 / C I R U .20001764$

Cir Cir. 2020;88(S1):112-115

Contents available at PubMed

www.cirugiaycirujanos.com

0009-7411/@ 2020 Academia Mexicana de Cirugía. Publicado por Permanyer. Este es un artículo open access bajo la licencia CC BY-NC-ND (http://creativecommons.org/licenses/by-nc-nd/4.0/).
} 


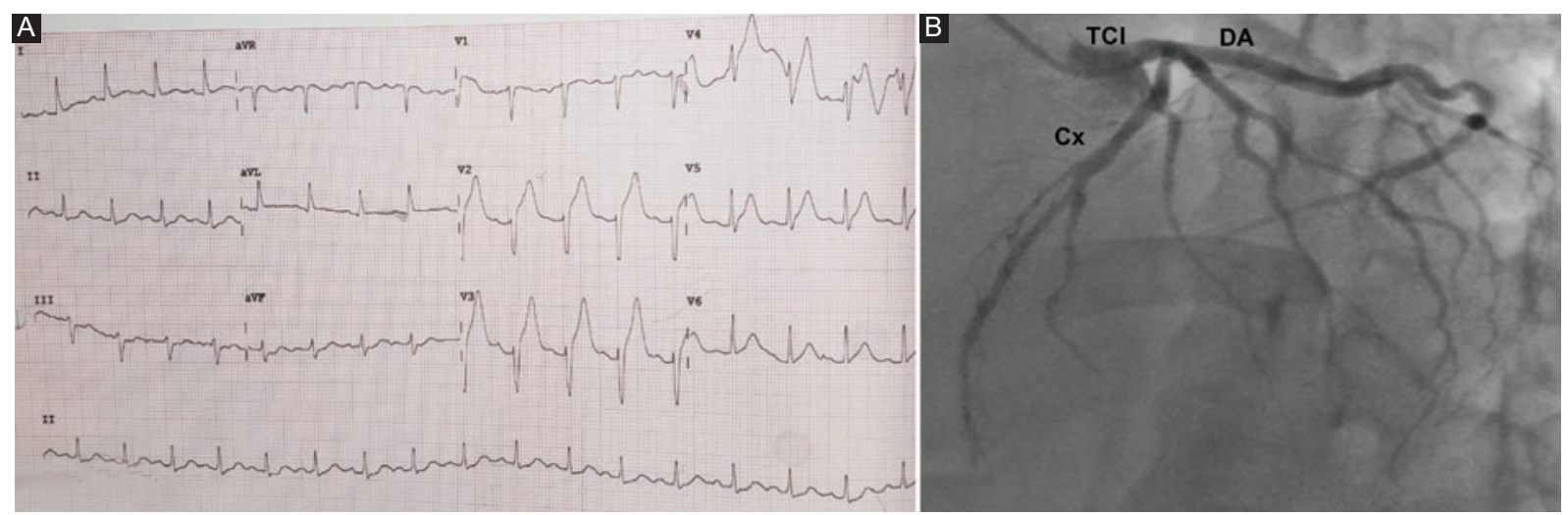

Figura 1. A: Electrocardiograma de 12 derivaciones, con elevación del segmento ST en la cara anterior por lesión subepicárdica anteroseptal. B: coronariografía. Proyección oblicua anterior derecha, tronco coronario izquierdo (TCl) corto, trifurcado; descendente anterior (DA): Gensini 3 , primera y segunda diagonal con lesión ostial no significativa, tercio distal con enfermedad difusa, flujo final TIMI 3; ramus intermedio sin lesiones angiográficas; circunfleja (Cx) sin lesiones.

empleado. En pacientes asintomáticos se pueden identificar anormalidades por ecocardiograma en la mitad de ellos. Al integrar cuadro clínico, electrocardiograma y ecocardiograma se han reportado alteraciones principalmente en el pericardio $(35 \%)$, el miocardio $(30 \%)$, las arterias coronarias $(12 \%)$ y las válvulas cardíacas $(6 \%)$, y de manera concomitante pericarditis y miocarditis en el $6 \%{ }^{3}$. Con resonancia magnética se evidencian alteraciones hasta en el $61 \%$ de los $\operatorname{casos}^{4}$, y en pacientes que presentan dolor precordial típico se demuestran lesiones por angiografía coronaria en el $70 \%{ }^{5}$.

La afección cardíaca es un factor de mal pronóstico, ya que incrementa los riesgos de recaída de la enfermedad, resistencia al tratamiento y mortalidad 6 .

\section{Caso clínico}

Se presenta el caso de un paciente de 49 años previamente sano, sin antecedentes de riesgo cardiovascular, quien inició con escleritis anterior difusa, descarga nasal purulenta de repetición, hipoacusia derecha y artralgias de pequeñas articulaciones, agregándose 5 meses después fiebre y parálisis facial periférica derecha. Fue valorado por el servicio de otorrinolaringología, que sospechó colesteatoma por imágenes de tomografía computada de oído medio. Se realizaron mastoidectomía y resección de oído medio derecho. Los estudios complementarios reportaron leucocitos $10,840 / \mathrm{mm}^{3}$, linfocitos $1,131 / \mathrm{mm}^{3}$, neutrófilos $9,000 / \mathrm{mm}^{3}$, hemoglobina $11.9 \mathrm{~g} / \mathrm{dl}$, hematocrito $37.3 \%$, volumen corpuscular medio $87.2 \mathrm{fl}$, concentración de hemoglobina corpuscular media $31.9 \mathrm{~g} / \mathrm{dl}$, urea $24 \mathrm{mg} / \mathrm{dl}$, creatinina $1.1 \mathrm{mg} / \mathrm{dl}$, glucosa $87 \mathrm{mg} / \mathrm{dl}$, colesterol total $193 \mathrm{mg} / \mathrm{dl}$, colesterol ligado a lipoproteínas de alta densidad $32 \mathrm{mg} / \mathrm{dl}$, colesterol ligado a lipoproteínas de baja densidad $149 \mathrm{mg} / \mathrm{dl}$, triglicéridos $168 \mathrm{mg} / \mathrm{dl}$; examen general de orina: densidad 1.010, pH 6.5, proteínas, nitritos y leucocitos negativos, sedimento urinario con 45-50 eritrocitos por campo con un $30 \%$ de eritrocitos dismórficos; velocidad de sedimentación globular $51 \mathrm{~mm} / \mathrm{h}$, proteína $C$ reactiva $12.4 \mathrm{mg} / \mathrm{dl}$, ANCA 1:320 y antiproteinasa $378 \mathrm{UR} / \mathrm{ml}$ (rango normal < $20 \mathrm{UR} / \mathrm{ml}$ ); exudado faríngeo, hemocultivo y urocultivo negativos, serología para virus de la inmunodeficiencia humana y para virus de la hepatitis B y $C$ negativa; radiografía de tórax sin alteraciones, y biopsia de oído medio con vasculitis y necrosis fibrinoide focal en pequeños vasos. En la valoración por reumatología se diagnosticó granulomatosis con poliangeítis de acuerdo con los criterios de clasificación del American College of Rheumatology de 19907.

Una semana posterior al diagnóstico el paciente presentó infarto agudo del miocardio con elevación del segmento ST, con dolor precordial típico, electrocardiograma de 12 derivaciones con taquicardia sinusal, frecuencia cardíaca de 108 lpm, P morfología +/- en V1, $0.1 \mathrm{mv}, 80 \mathrm{~ms}$, ap $+60^{\circ}$, PR $160 \mathrm{~ms}$, constante, R-R regular, QRS $80 \mathrm{~ms}$, aqrs $0^{\circ}$, ST desnivel positivo, V1 $0.1 \mathrm{mv}$, V2 $0.4 \mathrm{mv}$, V3 $0.3 \mathrm{mv}$, V4 $0.3 \mathrm{mv}$; onda T concordante con QRS, acuminada, máximo voltaje de 1.5 mv, qtc 465 ms, qr en DI y avl, rs en DIIl y avf, posición horizontal, dextrorrotado. Conclusión: taquicardia sinusal, bloqueo de fascículo anterior, lesión subepicárdica anteroseptal (Fig. 1 A). Creatina cinasa (CK) 1,295 U/l, CKMB $112 \mathrm{U} / \mathrm{l}$ y troponina I $9 \mathrm{ng} / \mathrm{ml}$. Ecocardiograma: acinesia anterolateral, apical, diafragmática y septal, 
hipocinesia posterobasal y fracción de eyección del ventrículo izquierdo $40 \%$. Fue trasladado a la unidad de cuidados coronarios y se realizó intervención coronaria percutánea por contraindicación para terapia de reperfusión farmacológica por la reciente cirugía de oído medio. La coronariografía reportó tronco trifurcado con lesión del $20 \%$ en segmento distal, descendente anterior tipo 3 de Gensini, segmento medio y distal con enfermedad difusa no significativa, primera y segunda diagonal con lesión ostial, flujo final TIMI 3; circunfleja sin lesiones angiográficas; coronaria derecha codominante sin lesiones (Fig. 1 B). No se encontró evidencia de oclusión coronaria por trombo o placa de ateroma, por lo que se consideró que los cambios se relacionaron con la actividad de la enfermedad. Inició antiagregación plaquetaria con aspirina y clopidogrel, estatina y heparina de bajo peso molecular, con mejoría de los síntomas, sin criterios de reperfusión.

El tratamiento de inducción a remisión de la granulomatosis con poliangeítis fue con pulsos de metilprednisolona y terapia biológica con rituximab. Egresó del hospital por mejoría y 3 meses después presentó un nuevo evento de dolor precordial sugestivo de síndrome isquémico coronario agudo con desenlace fatal. El puntaje pronóstico FFS (Five Factor Score) ${ }^{8}$ fue de 2 puntos, integrados por compromiso cardíaco y de sistema nervioso central, que predice una mortalidad del $46 \%$ a 5 años. El índice VDI (Vasculitis Damage Index $)^{9}$ fue de 7 por angina, infarto del miocardio, cardiomiopatía con infarto subsecuente y lesión de nervio craneal.

\section{Discusión}

En la granulomatosis con poliangeítis las alteraciones cardíacas son poco frecuentes; las manifestaciones clínicas incluyen dolor precordial, arritmias letales e insuficiencia cardíaca ${ }^{10}$. La mortalidad es del $26 \%$ después del primer año del diagnóstico ${ }^{11}$.

La vasculitis de las arterias coronarias es rara y presenta complicaciones como aneurismas, estenosis, trombosis y anormalidades en la microcirculación (arteriolas y vénulas), que a menudo son subclínicas en las primeras fases ${ }^{12}$.

Las alteraciones de las arterias coronarias conducen a hipoperfusión e isquemia, y se manifiestan clínicamente como angina e infarto agudo del miocardio. Los mecanismos fisiopatológicos son embolia coronaria, aortitis que provoca estenosis ostial coronaria y arteritis coronaria que genera disminución de la perfusión en arterias coronarias epicárdicas, arterias del miocardio y arteriolas ${ }^{13}$.

La arteritis coronaria es infrecuente en la granulomatosis con poliangeítis; sin embargo, en reportes de necropsias se describe como la alteración más frecuente, con una prevalencia del $50 \%$, encontrando en el reporte histopatológico vasculitis necrosante ${ }^{14}$.

Actualmente existen pocos reportes de casos de infarto agudo del miocardio en pacientes con granulomatosis con poliangeítis. En este caso presentamos un paciente con granulomatosis con poliangeítis e infarto agudo del miocardio secundario a arteritis coronaria como manifestación de la enfermedad.

\section{Conclusiones}

La granulomatosis con poliangeítis es una vasculitis sistémica con manifestaciones cardíacas poco frecuentes, e involucra pericarditis, miocarditis y arteritis coronaria como las tres primeras causas de afectación cardíaca. El diagnóstico es difícil de identificar, ya que los pacientes cursan asintomáticos de manera inicial. La arteritis coronaria y la vasculitis de arteriolas se manifiestan clínicamente como infarto agudo del miocardio, que puede desencadenar resultados fatales como en el caso presentado. Por ello, está indicado buscar estas manifestaciones clínicas intencionadamente con estudios de imagen para ofrecer tratamiento oportuno y así mejorar el pronóstico.

\section{Conflicto de intereses}

Los autores declaran no tener ningún conflicto de intereses.

\section{Responsabilidades éticas}

Protección de personas y animales. Los autores declaran que para esta investigación no se han realizado experimentos en seres humanos ni en animales.

Confidencialidad de los datos. Los autores declaran que han seguido los protocolos de su centro de trabajo sobre la publicación de datos de pacientes.

Derecho a la privacidad y consentimiento informado. Los autores han obtenido el consentimiento informado de los pacientes y/o sujetos referidos en el artículo. Este documento obra en poder del autor de correspondencia. 


\section{Bibliografía}

1. Hoffman GS, Kerr GS, Leavitt RY, Hallahan CW, Lebovics RS, Travis WD, et al. Wegener granulomatosis: an analysis of 158 patients. Ann Intern Med. 1992;116:488-98.

2. Ben Ghorbel I, Belfeki N, Baouendi N, Ben Salem T, Houman $M H$ Granulomatosis with polyangiitis in Tunisia. Reumatismo. 2017;69:23-9

3. Mcgeoch L, Carette S, Cuthbertson D, Hoffman GS, Khalidi N, Koening $\mathrm{CL}$, et al. Cardiac involvement in granulomatosis with polyangiitis. J Rheumatol. 2015;42:1209-12.

4. Pugnet $G$, Gouya $H$, Puéchal $X$, Terrier $B$, Kahan $A$, Legmann $P$, et al Cardiac involvement in granulomatosis with polyangiitis: a magnetic resonance imaging study of 31 consecutive patients. Rheumatology (Oxford). 2017;56:947-56.

5. Hazebroek MR, Kemna MJ, Schalla S, Sanders-van Wijk S, Gerretsen SC, Dennert R, et al. Prevalence and prognostic relevance of cardiac involvement in ANCA-associated vasculitis: eosinophilic granulomatosis with polyangiitis and granulomatosis with polyangiitis. Int $\mathrm{J}$ Cardiol. 2015;199:170-9.

6. Miszalski-Jamka T, Szczeklik W, Sokołowska B, Miszalski-Jamka K, Karwat K, Grządziel G, et al. Cardiac involvement in Wegener's granulomatosis resistant to induction therapy. Eur Radiol. 2011;21:2297-304.
7. Leavitt RY, Fauci AS, Bloch DA, Michel BA, Hunder GG, Arend WP, et al. The American College of Rheumatology 1990 criteria for the classification of Wegener's granulomatosis. Arthritis Rheum. 1990;33:1101-7.

8. Guillevin L, Pagnoux C, Seror R, Mahr A, Mouthon L, Le Toumelin P. The Five-Factor Score revisited: assessment of prognoses of systemic necrotizing vasculitides based on the French Vasculitis Study Group (FVSG) cohort. Medicine (Balt). 2011;90:19-27.

9. Exley AR, Bacon PA, Luqmani RA, Kitas GD, Gordon C, Savage CO, et al. Development and initial validation of the Vasculitis Damage Index for the standardized clinical assessment of damage in the systemic vasculitides. Arthritis Rheum. 1997;40:371-80.

10. Fauci AS, Wolff SM. Wegener's granulomatosis and related diseases. Dis Mon. 1977;23:1-36.

11. Flossmann O, Berden A, de Groot K, Hagen C, Harper L, Heijl C, et al. Long-term patient survival in ANCA-associated vasculitis. Ann Rheum Dis. 2011;70:488-94.

12. Jeon CH, Kim YK, Chun EJ, Kim JA, Yong HS, Doo KW, et al. Coronary artery vasculitis: assessment with cardiac multi-detector computed tomography. Int J Cardiovasc Imaging. 2015;31:59-67.

13. Korantzopoulos P, Papaioannides D, Siogas K. The heart in Wegener's granulomatosis. Cardiology. 2004;102:7-10.

14. Forstot JZ, Overlie PA, Neufeld GK, Harmon CE, Forstot SL. Cardiac complications of Wegener granulomatosis: a case report of complete heart block and review of the literature. Semin Arthritis Rheum. 1980;10:148-54. 